\title{
Infecções hospitalares relacionadas a procedimentos invasivos em unidades de terapia
}

\section{intensiva: revisão integrativa}

Nosocomial infections related to invasive procedures in intensive care units: integrative review

Infecciones hospitalarias relacionados con procedimientos invasivos en unidades de cuidados intensivos:

revisión integradora.

Marcos André Siqueira de Sousa ${ }^{1}$, Glícia Cardoso Nascimento ${ }^{1}$, Felipe Lazarini Bim², Layze Braz de Oliveira ${ }^{2}$, Adélia Dalva da Silva Oliveira ${ }^{3}$

1. Universidade Federal do Piauí. Programa de Pós-graduação em Enfermagem. Teresina, Piauí, Brasil

2. Escola de Enfermagem de Ribeirão Preto. Universidade de São Paulo, Ribeirão Preto, São Paulo, Brasil.

3. Centro Universitário UNINOVAFAPI, Mestrado Profissional em Saúde da Família, Teresina, Piauí, Brasil.

\section{ABSTRACT}

Objectives: to discuss the relationship between invasive procedures and hospital infections in Intensive Care Units from international scientific production. Method: integrative review, with a view to answer the following question: What is the relation between the use of long-term invasive procedures and the development of hospital infections in Intensive Care Units? It was carried out at the bases: PUBMED, Science Direct and Web of Science, considering the inclusion and exclusion criteria. Results: ten articles were selected. The nature of the procedure, the environment, the technique and the length of time the catheter was kept were highlighted as the main risk factors. The main devices related to the development of infections are urinary catheterization, endotracheal intubation, mechanical ventilation and intravascular catheters. Conclusion: There is still a shortage of studies that take a more in - depth look at the relationship between hospital infection and invasive procedures, especially with a simultaneous approach of all the invasive procedures inserted.

Descriptors: Intensive care unit; Infection; Hospital infection.

\section{RESUMO}

Objetivos: discutir a partir da produção cientifica internacional a relação entre procedimentos invasivos e infecções hospitalares em Unidades de Terapia Intensiva. Método: revisão integrativa, com vistas á responder a seguinte questão: Qual a relação entre uso de procedimentos invasivos de longa permanência e o desenvolvimento de infecções hospitalares em Unidades de Terapia Intensiva? Foi realizada nas bases: PUBMED, Science Direct e Web of Science, considerando-se os critérios de inclusão e exclusão. Resultados: foram selecionados dez artigos. A natureza do procedimento, o ambiente, a técnica e o tempo de permanência do cateter foram destacados como principais fatores de risco. Os principais dispositivos relacionados com o desenvolvimento de infecções são a cateterização urinária, intubação endotraqueal, ventilação mecânica e cateteres intravasculares. Conclusão: Ainda existe uma certa escassez de estudos que contemplem de forma mais aprofundada a relação infecção hospitalar e procedimentos invasivos, principalmente com abordagem simultânea de todos os procedimentos invasivos inseridos.

DESCRITORES: Unidades de terapia intensiva; Infecção; Infecção hospitalar.

\section{RESUMÉN}

Objetivos: discutir a partir de la producción científica internacional la relación entre procedimientos invasivos e infecciones hospitalarias en Unidades de Terapia Intensiva. Método: revisión integrativa, con miras a responder a la siguiente pregunta: ¿Cuál es la relación entre el uso de procedimientos invasivos de larga permanencia y el desarrollo de infecciones hospitalarias en Unidades de Terapia Intensiva? Se realizó en las bases: PUBMED, Science Direct y Web of Science, considerando los criterios de inclusión y exclusión. Resultados: se seleccionaron diez artículos. La naturaleza del procedimiento, el ambiente, la técnica y el tiempo de permanencia del catéter fueron destacados como principales factores de riesgo. Los principales dispositivos relacionados con el desarrollo de infecciones son la cateterización urinaria, intubación endotraqueal, ventilación mecánica y catéteres intravasculares. Conclusión: Aún existe una cierta escasez de estudios que contemplan de forma más profunda la relación infección hospitalaria y procedimientos invasivos, principalmente con abordaje simultáneo de todos los procedimientos invasivos insertados.

Descriptores: Unidade de terapia intensiva; infección; Infección hospitalaria.

\section{Como citar este artigo:}

Sousa MAS, Nascimeto GC, Bim FL, Oliveira LB, Oliveira ADS. Infecções hospitalares relacionadas a procedimentos invasivos em unidades de terapia intensiva: revisão integrativa. Rev Pre Infec e Saúde.2017;3(3):49-58. Available from: http://www.ojs.ufpi.br/index.php/nupcis/article/view/5848 


\section{INTRODUÇÃO}

As Infecções Relacionadas à Assistência a Saúde (IRAS), são reconhecidas por seu caráter multifatorial, já que são diretamente associadas a fatores intrínsecos e extrínsecos entre eles à gravidade da doença, as condições nutricionais dos pacientes, a natureza dos procedimentos diagnósticos ou terapêuticos, bem como ao tempo de internação. Estas trazem reflexos econômicos e sociais, visto que causam um aumento no tempo de internação do paciente, aumento da morbidade, e crescimento de organismos multirresistentes dentre outros ${ }^{1}$.

A prevalência de infecções hospitalares é maior em países subdesenvolvidos. Estudos recentes evidenciaram uma prevalência no Brasil de $22,8 \%$. Esta taxa revela-se ainda mais acentuada quando comparada a estudos em hospitais europeus os quais demonstram taxas de IH menores que $9 \%^{2-3}$.

No hospital são vários os ambientes susceptíveis a desenvolverem IRAS, dentre estes as Unidades de Terapia Intensiva (UTI), considerada área crítica, tanto pela instabilidade hemodinâmica dos pacientes internados, quanto pelo risco elevado de desenvolver IRAS. As UTIs são unidades direcionadas ao atendimento de pacientes em condições grave, que de uma forma geral carecem de monitoramento e suporte intensivos e contínuos de suas funções vitais ${ }^{4}$.

A prevalência das IRAS nas UTIs pode variar de 18 a 54\%, sendo de cinco a dez vezes maior neste ambiente que em outros setores de internação hospitalar. As taxas de mortalidades por este agravo revelam-se acentuadas chegando a $60 \%$ do total de óbitos neste ambiente ${ }^{5}$.

Pela complexidade do quadro clínico, pacientes internados em UTI estão naturalmente expostos a uma maior quantidade de procedimentos invasivos, cirurgias de maior complexidade e uso de grande quantidade de drogas, tais como as imunossupressoras, o que favorece o desenvolvimento de micro-organismos e, por conseguinte, a colonização e/ou infecção por micro-organismos, inclusive multirresistentes.

0 uso de procedimentos invasivos, principalmente neste ambiente contribui significativamente para a ocorrência da infecção, justificando a existência de diversas topografias infecciosas (urinária, respiratória, sitio cirúrgico, corrente sanguínea).

Este cenário, reforça a relevância deste estudo para toda a área da saúde, em vistas a associação já comprovada entre uso de procedimentos invasivos e desenvolvimento de Infecções Relacionados à Assistência à Saúde, em pacientes internados em $\mathrm{UTI}^{5}$.

Baseado nesta problemática, o presente estudo teve como objetivo realizar uma revisão integrativa da literatura sobre infecções hospitalares em UTI relacionada a procedimentos invasivos, e elencar possíveis fatores de risco para o desenvolvimento da mesma.

\section{MÉTODOS}

Trata-se de uma revisão integrativa (RI) da literatura, importante ferramenta da prática baseada em evidências. A escolha do método se deu pela possibilidade de reunir e sintetizar resultados de múltiplos estudos publicados sobre delimitada temática de maneira sistemática e ordenada, contribuindo para o aprofundamento do tema de pesquisa.

O estudo foi operacionalizado a partir das etapas seguintes: elaboração da questão norteadora da pesquisa; busca na literatura cientifica por estudos primários; extração de dados; avaliação dos estudos primários; análise e síntese dos resultados e apresentação da revisão. A questão norteadora para a condução da RI foi elaborada por meio da estratégia PICO (P: Paciente, I: Intervenção, C: Comparação e O: 
Outcomes ou desfecho): Qual o perfil das infecções hospitalares relacionadas a procedimentos invasivos em Unidades de Terapia Intensiva?'

Para elaboração da pesquisa foi realizada uma busca nas bases de dados: Medical Literature Analysis and Retrieval Systemon-line (Medline); Science Citation Index Expanded (Web of Science) e SciVerse Science Direct (Elsevier), utilizando-se a combinação de descritores controlados, aqueles estruturados e organizados para facilitar o acesso à informação e que estejam cadastrados nos Medical Subject Headings (MesH): “Intensive care unit [and] nosocomial infection", "Intensive care unit [and] invasive procedures" e "nosocomial infection [and] invasive procedures".

Os critérios de inclusão delimitados para a presente revisão foram: estudos primários que abordassem a temática das infecções hospitalares em UTI e sua relação com o desenvolvimento de procedimentos invasivos; estar disponível na integra de forma eletrônica e gratuita e que compreendessem a faixa temporal proposta para o estudo de 2006 a 2016. Como critério de exclusão não se utilizou artigos que abordassem UTIs neonatais ou pediátricas, artigos que se repetissem nas bases de dados, artigos de opinião, artigos de reflexão e editoriais.

$\mathrm{Na}$ etapa seguinte, os estudos foram avaliados quanto ao ano, idioma, metodologia e aplicabilidade do resultado na prática, rigor metodológico dos estudos, as intervenções mensuradas e os resultados encontrados, tipo de estudo e o nível de evidência, considerando: 1 revisões sistemáticas ou metanálise de relevantes ensaios clínicos; 2 - evidências de pelo menos um ensaio clínico randomizado controlado bem delineado; 3 - ensaios clínicos bem delineados sem randomização; 4 - estudos de coorte e de casocontrole bem delineados; 5 - revisão sistemática de estudos descritivos e qualitativos; 6 - evidências derivadas de um único estudo descritivo ou qualitativo; 7 - opinião de autoridades ou comitês de especialistas incluindo interpretações de informações não baseadas em pesquisas ${ }^{7}$.

A análise e síntese dos estudos primários foram realizadas na forma descritiva, possibilitando ao leitor uma síntese de cada estudo incluído na RI e comparações, enfatizando as diferenças e similaridades entre os estudos.

\section{RESULTADOS}

Dos quarenta e um (41) artigos selecionados, nove (09) foram excluídos por repetirem-se nas bases de dado, enquanto outros vinte e dois (22) não se correlacionavam com os objetivos da revisão integrativa, restando assim dez (10) artigos que atendessem a todos os critérios de inclusão da pesquisa, verificou-se ainda que todos foram publicados no idioma inglês.

A figura 1 apresenta a síntese dos estudos primários incluídos na RI analisados quanto ao ano, base de dados e periódico da publicação. Com relação à indexação das publicações relacionadas, houve predomínio da base de dados PUBMED. O periódico mais relacionado às divulgações das pesquisas foi o Journal of Hospital Infection.

$\mathrm{Na}$ figura 2 evidencia-se a relação entre as infecções hospitalares ocasionadas nas UTIs tendo como fatores de risco os procedimentos invasivos aos quais os pacientes foram submetidos.

De uma forma geral os estudos relataram alta prevalência de infecções nas UTI com uma associação marcante com procedimentos invasivos, sendo estes considerados fatores de risco para o desenvolvimento de IRAS em UTIs. Quanto aos tipos de IHs, percebe-se o predomínio da pneumonia associada à ventilação mecânica, seguida por infecções de trato urinário, infecções de sítio cirúrgico e tecidos moles. 


\begin{tabular}{|c|c|c|c|c|}
\hline $\begin{array}{l}\text { Ordem } \\
\text { dos } \\
\text { Artigos }\end{array}$ & $\begin{array}{c}\text { Base de } \\
\text { dado/Ano }\end{array}$ & Título do artigo & Periódico & $\begin{array}{l}\text { Nivel de } \\
\text { evidência }\end{array}$ \\
\hline $\mathrm{A} 1$ & PUBMED/2006 & $\begin{array}{l}\text { Nosocomial infections in the } \\
\text { intensive care unit of a gynecology } \\
\text { clinic7 }\end{array}$ & Med Pregl & IV \\
\hline A2 & PUBMED/2006 & $\begin{array}{l}\text { Hospital-acquired } \\
\text { surveillance in a } \\
\text { intensive care unit8 }\end{array}$ & $\begin{array}{l}\text { Journal of } \\
\text { Hospital } \\
\text { Infection }\end{array}$ & IV \\
\hline A3 & PUBMED/2008 & $\begin{array}{l}\text { Frequency, pattern and etiology of } \\
\text { nosocomial infection in intensive } \\
\text { care unit: an experience at a } \\
\text { tertiary care hospital } 9\end{array}$ & $\begin{array}{l}\text { Journal Ayub } \\
\text { Med Coll } \\
\text { Abbottabad. }\end{array}$ & IV \\
\hline $\mathrm{A} 4$ & $\begin{array}{c}\text { Science } \\
\text { direct } / 2007\end{array}$ & $\begin{array}{l}\text { The first prevalence survey of } \\
\text { nosocomial infections in the } \\
\text { University Hospital Centre 'Mother } \\
\text { Teresa' of Tirana, Albania10 }\end{array}$ & $\begin{array}{l}\text { Journal of } \\
\text { Hospital } \\
\text { Infection }\end{array}$ & IV \\
\hline $\mathrm{A} 5$ & $\begin{array}{c}\text { Science } \\
\text { direct }\end{array}$ & $\begin{array}{l}\text { Impact of hospital-wide infection } \\
\text { rate, invasive procedures use and } \\
\text { antimicrobial consumption on } \\
\text { bacterial resistance inside an } \\
\text { intensive care unit11 }\end{array}$ & $\begin{array}{l}\text { Journal of } \\
\text { Hospital } \\
\text { Infection }\end{array}$ & IV \\
\hline A6 & $\begin{array}{c}\text { Web of } \\
\text { Science/2010 }\end{array}$ & $\begin{array}{l}\text { Nosocomial Infection in an } \\
\text { Intensive Care Unit in a Brazilian } \\
\text { University Hospital12 }\end{array}$ & $\begin{array}{l}\text { Rev. Latino-Am. } \\
\text { Enfermagem }\end{array}$ & IV \\
\hline A7 & $\begin{array}{c}\text { Web of } \\
\text { Science/2013 }\end{array}$ & $\begin{array}{l}\text { Comparison of intensive-care-unit- } \\
\text { acquired infections and their } \\
\text { outcomes among patients over and } \\
\text { under } 80 \text { years of age } 13\end{array}$ & $\begin{array}{c}\text { Doenças } \\
\text { Infecciosas e } \\
\text { Microbiologia } \\
\text { Clínica }\end{array}$ & IV \\
\hline A8 & PUBMED/2013 & $\begin{array}{l}\text { The prevalence of nosocomial and } \\
\text { community acquired infections in a } \\
\text { university hospital: an } \\
\text { observational study } 14\end{array}$ & $\begin{array}{l}\text { Dtsch Arztebl } \\
\text { Int. }\end{array}$ & IV \\
\hline A9 & $\begin{array}{c}\text { Science } \\
\text { Direct/2014 }\end{array}$ & $\begin{array}{l}\text { Nosocomial infection } \\
\text { characteristics in a burn intensive } \\
\text { care unit: Analysis of an eleven- } \\
\text { year active surveillance }\end{array}$ & Burns & IV \\
\hline $\mathrm{A} 10$ & $\begin{array}{l}\text { Web of } \\
\text { Science } \\
2015\end{array}$ & $\begin{array}{l}\text { Risk Factors of Carbapenem- } \\
\text { Resistant Klebsiella pneumoniae } \\
\text { Infection: A Serious Threat in ICUs }\end{array}$ & $\begin{array}{c}\text { Medical Science } \\
\text { Monitor }\end{array}$ & IV \\
\hline
\end{tabular}

Figura 1 - Detalhamento dos artigos analisados de acordo com a base de dados, ano de publicação, título do artigo, revistas da publicação e nível de evidência. Teresina (PI), 2017. 


\begin{tabular}{cl} 
Ordem dos & \multicolumn{1}{c}{ Objetivos do estudo } \\
Artigos & \\
A1 & Identificar os microorganismos mais frequentes causadores de infecções \\
& nosocomiais em Unidade de Terapia Intensiva em um Instituto de \\
& Ginecologia e Obstetrícia, Centro Clínico da Sérvia, no período de todos \\
& os pacientes foram tratados cirurgicamente, entubado e assistido por \\
& ventilação mecânica ${ }^{7}$.
\end{tabular}

A2 Identificar Caracteristicas demográficas, de origem do paciente, diagnóstico, escore de gravidade, doenças de base, procedimentos invasivos, microorganismos isolados e suscetibilidades aos antibióticos ${ }^{\natural}$.

A3 Avaliar a frequência de infecção hospitalar em pacientes internados em UTI e determinar os fatores etiológicos em tais pacientes".

A4 Estabelecer o padrão de uso de drogas antibacterianas, e sua associaçã̃o com indicadores de infecção hospitalar, em uma UTI de adultos nãoespecializada ${ }^{10}$.

A5 Relacionar o consumo microbiano entre procedimentos invasivos e micro resistência em uma UTI de um hospital universitário no sul do Brasil ${ }^{11}$.

A6 Determinar a incidência da infecção hospitalar (IH) em uma UTI, sua associação com características clínicas do paciente e sítios de ocorrência ${ }^{12}$.

A7 Avaliar a frequência e as consequências da $\mathrm{IH}$ adquirida em unidade de terapia intensiva, em pacientes idosos $\geq 80$ anos internados em UTI com um alto índice de procedimentos invasivos, e compararam estes resultados com os de pacientes mais jovens ${ }^{13}$.

A8 Avaliar a prevalência de $\mathrm{IH}$ em instituições particulares bem como a relaçẫo entre a duração da infecçẫo e fatores de risco ${ }^{14}$.

A9 Descrever as taxas de infecçắo nosocomial (NI), fatores de risco, os agentes etiológicos, susceptibilidade a antibióticos, a utilização do dispositivo invasivo e dispositivo associado taxas de infecção invasiva em uma unidade de queimados de terapia intensiva (UTI), na Turquia.

A10 Investigar os fatores de risco para infecçoues por Carbapenem Klebsiella pneumoniae (CRKP).

Figura 2 - Detalhamento dos artigos analisados de acordo com objetivos dos estudos. Teresina (PI), 2017 


Ordens dos
Artigos
A1

Os resultados constataram a presença de infecção, os agentes causadores mais isolados do tubo endotraqueal foram: Staphylococcus aureus, Staphylococcus spp coagulase negativo e Pseudomonas spp. Ao passo que a Escherichia coli e Enterococcus foram isolados a partir das feridas. O artigo correlaciona os procedimentos invasivos como fatores de risco ${ }^{7}$.

A equipe de controle de infecção recolheu os seguintes dados: características demográficas, diagnóstico, escore de gravidade, doenças de base, procedimentos invasivos, micro-organismos isolados e suscetibilidades aos antibióticos. Houve predomínio das pneumonias e infecções da corrente sanguínea. Uso elevado de procedimentos invasivos estatisticamente relacionados ao desenvolvimento de $\mathrm{IH}$ $(p<0,01)$. Foram registradas altas taxas de óbitos $(18,9 \%)^{8}$.

3 A frequência de infecção hospitalar foi de $29,13 \%$. Houve predomínio da infecção do trato urinário $(39,1 \%)$, seguido pela Infecção do trato respiratório (30,1\%). A pesquisa levantou o uso de procedimentos invasivos como fator de risco para o desenvolvimento de IH na UTI, ressaltando que pacientes internados em UTI estão em maior risco de adquirir IRAS a partir de diferentes fontes ${ }^{9}$.

Os resultados apontam elevados fatores de risco em UTI $(31,6 \%)$ em comparação com outras unidades, os principais fatores de risco elencados são: idade> 40 anos, o tempo de internação, diagnóstico de "trauma" na admissão e dispositivos invasivos ${ }^{10}$.

Foi identificada multirresistência bacteriana em UTI, em 31,3\% dos isolados. Durante o período estudado, 36,9\% ( $P<0,001)$ e 34,5\% ( $\quad<0,01)$ da taxa de multirresistência na UTI foi associada com o uso de procedimentos invasivos. Taxas de multirresistência em UTI são influenciadas pelos procedimentos invasivos em todo o hospital e consumo de antibióticos, dentro e fora da UTI ${ }^{11}$.

O estudo identificou que a taxa de IRAS na UTI foi de 20,3\%. A permanência média foi de 19,3 dias para pacientes com IH e 20,2 dias para colonizados com micro-organismos resistentes, o uso de dispositivos invasivos foram significativamente associados com a ocorrência de infecção em UTI ${ }^{12}$.

Houve alta prevalência de IRAS $(16,5 \%)$ na UTI em pacientes idosos, os quais necessitaram de maior uso de procedimentos invasivos, em comparação a pacientes jovens. Um modelo de Cox identificou cateterismo venoso central e ventilação mecânica invasiva por mais de dois dias como fatores de risco independentes para a IH em UTI. As associações entre IH e permanência prolongada na UTI, o aumento da carga de trabalho de enfermagem, e as taxas de mortalidade na UTI e hospitalar foram similares em pacientes jovens e idosos ${ }^{13}$.

A prevalência de infecção geral na UTI foi de $11,2 \%$. As IH mais comuns foram às infecções de sítio cirúrgico (29\%), trato respiratório (19\%) e infecções do trato urinário (16\%). Os patógenos mais comuns foram Escherichia coli, estafilococos coagulasenegativo, Enterococcus spp, e Pseudomonas aeruginosa. A análise de regressão multivariada revelou o uso de dispositivo invasivo como fator de risco independente para $(O R$ de 1,4). Outros fatores de risco incluíram idade, tempo de internação atual ou anterior, trauma, internação em uma UTI e ventilação artificial ${ }^{14}$.

Neste estudo, a análise univariada sugeriu que a superfície total queimada (TBSA), queimadura cheia de espessura, idade, lesão inalatória, o tempo médio de permanência hospitalar, transferência de outro hospital e índice do tempo médio de permanência hospitalar como fatores de risco potenciais para aquisição de NI. O número total de casos com o NI foi 469 e apesar dos sinais NI clínicos significativos, 87 deles tiveram resultados negativos de cultura. P. aeruginosa (241), Acinetobacter baumannii (186) e Staphylococcus aureus (69) foram as bactérias isoladas mais comuns. No estudo as taxas NI associados ao cateter vascular central (75\%), cateter urinário (25\%)e utilização do ventilador (90\%), demonstraram que a presença de dispositivo invasivo é um fator de risco para NI.

O estudo mostrou na análise univariada como fatores de risco para a infecção por Carbapenem-resistant Klebsiella pneumoniae (CRKP), a Imunossupressão (OR: 2,186), sonda nasogástrica (OR: 3,562), cateter arterial periférica (OR: 2,545), e sendo admitido na unidade de neurocirurgia (OR: 4,324). A análise multivariada revelou o uso de cefalosporinas de terceira geração (OR: 4,699), o uso de sonda nasogástrica (OR: 3,983 ), e sendo internados em UTI neurocirúrgica (OR: 4,603) como fatores de risco independentes.

Figura 3. Detalhamento dos artigos analisados de acordo com os principais resultados dos estudos. Teresina (PI),2017. 


\section{DISCUSSÃO}

A assistência na UTI é constantemente desafiada por infecções relacionadas ao uso de procedimentos invasivos. A literatura pesquisada evidencia que o uso destes procedimentos pode contribuir na ocorrência de infecção, pela variedade de sítios em que são necessários aliado ao grande tempo de permanência e a ocorrência de bactérias multirresistentes ${ }^{1,7-14}$.

Por seu caráter reconhecidamente multifatorial, as IRAS são diretamente associadas a fatores intrínsecos e extrínsecos entre eles a gravidade da doença, as condições nutricionais dos pacientes, a natureza dos procedimentos diagnósticos ou terapêuticos, bem como ao tempo de internação ${ }^{7-11}$.

Dentre os fatores extrínsecos, o ambiente em que esse paciente se encontra, associado a fatores ligados a assistência são cruciais para o desenvolvimento de IRAS. Pacientes internados em UTI, devido à complexidade do quadro clínico, estão expostos a uma maior quantidade de procedimentos diagnósticos, cirurgias de maior complexidade e grande quantidade de drogas, tais como imunossupressoras, que em determinadas situações exigem extrapolar as barreiras naturais da pele ou simplesmente a sua mobilização pelos profissionais da saúde. Por estes motivos, estão sujeitos a riscos de 5 a 10 vezes maiores de adquirir infecção que aqueles de outras unidades de internação do hospital ${ }^{8-14}$.

Soma-se a isto o frequente uso de procedimentos invasivos para a manutenção da vida do paciente, principalmente aqueles de longa permanência com destaque para a cateterização urinária, a intubação traqueal, a ventilação mecânica, os cateteres intravasculares e equipamentos para suporte de vida ${ }^{8-13,15}$.

Estudo de Pottier et al. ${ }^{16}$, por meio de análise univariada revelou que elevado número de procedimentos invasivos foi significativamente associado com o risco de ocorrência de eventos adversos nas UTIs abordadas, com predomínio de eventos relacionados a causas infecciosas, sobretudo aqueles com causas mecânicas. 0 autor encontrou que $34,7 \%$ dos pacientes submetidos a algum procedimento invasivo havia experimentado ao menos um tipo de evento adverso, dos quais $11 \%$ destes vieram a óbito. Assim, a ocorrência de eventos adversos relacionados a procedimentos invasivos é uma questão importante na UTI uma vez que carrega consequências clínicas potencialmente graves, incluindo a morte.

Além disto, pela pouca quantidade de Unidades de Terapia Intensiva, principalmente em países em desenvolvimento, como o Brasil, o aumento do tempo de internação por agravos relacionados a assistência a saúde atribui as IRAS forte fator social ao limitar o uso da mesma a outro usuário que necessite do acesso a terapia intensiva $^{14,17-18}$.

Com relação aos tipos de procedimentos invasivos, a cateterização urinaria destaca-se pela perceptível relação entre o tempo de permanência do cateter vesical de demora e o desenvolvimento de infecção de trato urinário, uma das infecções mais comuns e importantes em ambiente hospitalar, sendo a duração da cateterização vesical um importante fator de risco reconhecido9. 11.

Outro preditor de alta mortalidade hospitalar em todo o mundo são as infecções relacionadas ao cateter venoso central. A infecção da corrente sanguínea relacionada a cateter ocorre quando o micro-organismo presente no local de inserção cai na corrente sanguínea e assim provoca uma infecção com grave comprometimento clínico, podendo resultar em septicemia. Os cateteres intravasculares, em especial os venosos, são dispositivos bastante utilizados na terapia intensiva para administração de medicamentos, soluções hidroeletrolíticas, sangue, e também para monitoramento dos padrões fisiológicos. Com a 
Sousa MAS et al

presença destes no sistema venoso profundo, o risco de infecção torna-se elevada ${ }^{10-13}$.

No entanto, entre as diversas topografias a mais frequente dentro da UTI é a Pneumonia Associada à Ventilação Mecânica, intimamente relacionada a intubação endotraqueal e instituição da ventilação mecânica invasiva. Pacientes sobre ventilação mecânica costumam estar em estado grave, sendo ainda mais susceptíveis a sofrerem inoculação de microrganismos por aspiração ou por aerossóis. Assim, procedimentos simples como, mudança de decúbito ou elevar a cabeceira do leito do paciente, pode provocar a passagem do liquido contaminado para o trato respiratório. Além disto, na maioria das instituições de saúde a troca do circuito ventilatório não é realizada no tempo preconizado, o que pode favorecer a colonização e posterior infecção ${ }^{5}$.

Com base nisso acredita-se que é prudente limitar o tempo de determinados procedimentos invasivos como a ventilação mecânica e o cateter (vesical e venoso) como medida de prevenção da IRAS de forma a diminuir o risco de pneumonia, infecção urinária e de corrente sanguínea de origem hospitalar em UTI's $s^{5,10-14}$.

Apesar da relação procedimentos invasivos e IRAS já ser consolidada, inclusive na literatura internacional ${ }^{8-15}$, esta revisão revelou uma certa escassez de estudos sobre o tema, principalmente no que concerne a avaliar simultaneamente o papel de cada procedimento invasivo inserido no paciente e sua relação no desenvolvimento da infecção, uma vez que em UTIs, é bem comum um paciente submeter-se á mais de um procedimento invasivo ao mesmo tempo.

Percebeu-se também, uma forte concentração de estudos de abordagem biomédica. No entanto, estudos de abordagem biopsicosocial são importantes no entendimento e na elaboração de medidas de prevenção e controle de IRAS nas UTIs, uma vez que as taxas de infecção hospitalar tem avançado conforme os avanços da tecnologia invasiva, e o conhecimento dos profissionais a

\section{Infecções hospitalares em UTI}

cerca do tema parece não ter acompanhado a velocidade de tal avanço. 0 que se percebe nos hospitais são incipientes ações de prevenção e controle das infecções por partes destes profissionais, que paradoxalmente são os responsáveis por fornecer uma assistência segura aos pacientes.

Assim acredita-se ser necessário mais estudos sobre o tema, para que este conhecimento possa contribuir no esclarecimento do acometimento de infecções em Unidades de Terapia Intensiva, destacando fatores de riscos associados às infecções, para que com isso possam ser elaboradas estratégias de prevenção de agravos relacionados as IRAS.

\section{CONCLUSÃO}

A prevalência de infecções hospitalares em Unidades de Terapia Intensiva relacionadas a procedimentos invasivos é elevada, preocupante e acompanhada de morbidade e mortalidade em níveis alarmantes. $\mathrm{Na}$ terapia intensiva a necessidade de utilizar procedimentos invasivos é uma constante necessária para a manutenção da vida, no entanto, ressalta-se que esta atividade deva ser feita com extrema prudência pelo profissional, respeitando as técnicas de assepsia, tempo de manutenção e outros cuidados de forma a evitar a contaminação do paciente pelo dispositivo.

Os principais dispositivos relacionados com o desenvolvimento de IRAS são a cateterização urinária, a intubação endotraqueal, a ventilação mecânica e os cateteres intravasculares, associados a diversas topografias. Ainda existe uma certa escassez de estudos que contemplem de forma mais aprofundada a relação IRAS e procedimentos invasivos, principalmente com abordagem simultânea de todos os procedimentos invasivos inseridos. Também ressalta-se a necessidade de uma maior abordagem do profissional responsável pela assistência. Assim, 
Sousa MAS et al

conclui-se que a importância relativa dos eventos adversos em termos de impacto, morbidade e mortalidade neste ambiente ainda é mal avaliada.

Conclui-se então que os avanços tecnológicos dos procedimentos invasivos, diagnósticos e terapêuticos, e o aparecimento cada vez mais comum de micro-organismos multiresistentes aos antimicrobianos tornaram as infecções em UTI um importante problema de saúde pública se tornando em um desafio para os profissionais de saúde na busca pela excelência na assistência.

\section{REFERÊNCIAS}

1. Sousa ÁFL, Queiroz AAFLN, Oliveira LB, Valle ARMC, Moura MEB. Representações sociais da infecção comunitária por profissionais da atenção primária. Acta paul enferm. [Internet]. 2015 Aug [cited 2016 Jan 09] ; 28( 5 ): 454-459. Available from:

http: / / www.scielo.br/scielo.php?script=sci_arttext \&pid=S0103-21002015000500454\&lng=en. http: / /dx.doi.org/10.1590/1982-0194201500076.

2. Gray J, Omar N. Nosocomial infections in neonatal intensive care units in developed and developin countries: how can we narrow the gap? J Hosp Infect. 2013; 83:193-5. http://dx.doi.org/doi:10.1016/j.jhin.2012.12.006

3. Romanelli RMC, Anchieta LM, Mourão MVA, Campos FA, Loyola FC, Jesus LA et al . Infecções relacionadas à assistência a saúde baseada em critérios internacionais, realizada em unidade neonatal de cuidados progressivos de referência de Belo Horizonte, MG. Rev. bras. epidemiol. 2013 Mar; $16(1)$ ): 77-86. Available from: http: / / www.scielo.br/scielo.php?script=sci_arttext \&pid=S1415-790X2013000100077\&lng=en.

http://dx.doi.org/10.1590/S1415$790 \times 2013000100008$.

4. Oliveira AC, Oliveira PA, Iquiapaza RA, Lacerda ACS. Infecções relacionadas à assistência em saúde e gravidade clínica em uma unidade de terapia

\section{Infecções hospitalares em UTI}

intensiva. Rev Gaúcha Enferm. 2012 Sep; 33(3): 89-96. Available from: http://www.scielo.br/scielo.php?script=sci_arttext \&pid=S1983-14472012000300012\&lng=en. http://dx.doi.org/10.1590/S198314472012000300012.

5. Batista OMA, Santos MSG, Alexandria FED, Madeira MZA, Valle ARMC, Sousa AFL. Sensitivity of embryos related to the pneumonia associated with the ventilation mechanics. $R$ pesq cuid fundam online 2013;5(6):224-33. Available from: https: //dialnet.unirioja.es/servlet/articulo?codigo $=5091141$

6. Mendes KDS, Silveira RCCP, Galvão CM. Revisão integrativa: método de pesquisa para a incorporação de evidências na saúde e na enfermagem. Textocontexto - enferm [Internet]. 2008 Oct/Dec [cited 2013 June 15];17(4):758-64. Available from: http: / /www.scielo.br/scielo.php?script=sci_arttext \&pid=S010407072008000400018\&lng=en\&nrm=iso

7. Melnyk BM, Fineout-Overholt E. Making the case for evidence-based practice. In: Melnyk BM, Fineout-Overholt E. Evidence-based practice in nursing \& healthcare: a guide to best practice. Philadelphia: Lippincot Williams \& Wilkins; 2005 [Internet]. 2006 [cited 2013 Aug3];3-24. Available from:

http://download.lww.com/wolterskluwer_vitalstre am_com/PermaLink/NCNJ/A/NCNJ_546_

156_2010_08_23_SADFJO_165_SDC216.pdf

8. Arsenijevic, L, Popovic, N, Gojnic, M. Nosocomial infections in the intensive care unit of a gynecology clinic. Medicinskipregled. 2006; MarApr;59(3-4):165-8. Available from: http://www.ncbi.nlm.nih.gov/pubmed/17066590.

9. Orsi GB, Scorzolini L, Franchi C, Mondillo V, Rosa G, Venditti M. Hospital-acquired infection surveillance in a neurosurgical intensive care unit. J Hosp Infect. 2006 Sep;64(1):23-9. Available from: http://www.ncbi.nlm.nih.gov/pubmed/16839643. 10. Shaikh JM, Devrajani BR, Shah SZ, Akhund T, Bibi I. Frequency, pattern and etiology of 
Sousa MAS et al

nosocomial infection in intensive care unit: an experience at a tertiary care hospital. J Ayub Med Coll Abbottabad. 2008 Oct-Dec;20(4):37-40. Available

from: http://www.ncbi.nlm.nih.gov/pubmed/19999200.

11. Faria S, Sodano L, Gjata A, Dauri M, Sabato AF, Bilaj A, Mertiraj O, Llazo E, Kodra Y, Schinaia N; PrevalenceStudyGroup. The first prevalence survey of nosocomial infections in the University Hospital Centre 'Mother Teresa' of Tirana, Albania. J Hosp Infect. 2007 Mar;65(3):244-50. Available from: http://www.ncbi.nlm.nih.gov/pubmed/17241694.

12. Jacoby TS, Kuchenbecker RS, Dos Santos RP, Magedanz L, Guzatto P, Moreira LB. Impact of hospital-wide infection rate, invasive procedures use and antimicrobial consumption on bacterial resistance inside an intensive care unit. J Hosp Infect. $2010 \quad$ May;75(1):23-7. doi: 10.1016/j.jhin.2009.11.021. Available from: http://www.ncbi.nlm.nih.gov/pubmed/20338669.

13. Oliveira AC, Kovner CT, da Silva RS. Nosocomial infection in an intensive care unit in a Brazilian university hospital. Rev Lat Am Enfermagem. 2010 Mar-Apr;18(2):233-9. Available from: http://www.ncbi.nlm.nih.gov/pubmed/20549123.

14. Maillet JM, Guérot E, Novara A, Le Guen J, Lahjibi-Paulet H, Kac G, Diehl JL, Fagon JY. Comparison of intensive-care-unit-acquired infections and their outcomes among patients over and under 80 years of age. J Hosp Infect. 2014 Jul;87(3):152-8. doi: 10.1016/j.jhin.2014.03.011. Available from:

\section{Infecções hospitalares em UTI}

15. Ott E, Saathoff S, Graf K, Schwab F, Chaberny IF. The prevalence of nosocomial and community acquired infections in a university hospital: an observational study. DtschArztebl Int. 2013 Aug;110(31-32):533-40. doi: 10.3238/arztebl.2013.0533. Available from: http://www.ncbi.nlm.nih.gov/pubmed/24069074.

16. Pottier V, Daubin C, Lerolle N, Gaillard C, Viquesnel G, Plaud B, Hanouz JL, Charbonneau P. Overview of adverse eventsrelated to invasiveprocedures in theintensivecareunit. Am J Infect Control. 2012 Apr;40(3):241-6. doi: 10.1016/j.ajic.2011.04.005. Available from: http://www.ncbi.nlm.nih.gov/pubmed/21813208.

17. Nangino GO, Oliveira CD, Correia PC, Machado NM, Dias ATB. Impacto financeiro das infecções nosocomiais em unidades de terapia intensiva em hospital filantrópico de Minas Gerais. Rev. bras. ter. intensiva. 2012 Dec; 24( 4 ): 357-361. Availablefrom:

http: / / www.scielo.br/scielo.php?script=sci_arttext \&pid=S0103-507X2012000400011\&lng=en. http://dx.doi.org/10.1590/S0103-

507X2012000400011.

18. Vilarinho LM, Vilarinho MLCM, Silva FL, Guimaraes MSO, Leal ACAM. Isolamento de staphylococcus aureus em mãos de profissionais de Unidades de terapia Intensiva. Rev. Pre. Infec e Saúde, 2015; (1):1: 10-18. Available from: www.ojs.ufpi.br/index.php/nupcis/article/downlo $\mathrm{ad} / 3421 / \mathrm{pdf}$

http://www.ncbi.nlm.nih.gov/pubmed/24856114.

\section{COLABORAÇÕES}

Sousa MAS, Nascimeto GC, Bim FL, Oliveira LB e Oliveira ADS contribuíram na coleta e interpretação dos resultados obtidos e ainda, na elaboração e organização das ideias e nas revisões sucessivas até a aprovação final.

\section{CONFLITOS DE INTERESSE}

Não há conflitos de interesse a declarar

\section{CORRESPONDENCIA}

Marcos André Siqueira de Sousa

Universidade Federal do Piauí

Campus Universitário Ministro Petrônio Portella, s/n - Ininga, Teresina - PI, 64049-550

E-mail: marcosandrs4@gmail.com 